\title{
Direct evidence of a graded magnetic interface in bi-magnetic core/shell nanoparticles using electron magnetic circular dichroism \\ (EMCD)
}

Daniel del-Pozo-Bueno ${ }^{1,2, *}$, Maria Varela ${ }^{3, *}$, Marta Estrader ${ }^{4,2}$, Alberto López-Ortega ${ }^{5,6, *}$, Alejandro G. Roca ${ }^{7,}$, , Josep Nogués ${ }^{7,8}$, Francesca Peiró ${ }^{1,2}$, Sònia Estradé ${ }^{1,2}$

${ }^{1}$ LENS-MIND, Dept. Enginyeries Electrònica i Biomèdica, Universitat de Barcelona, Martí i Franques 1, E-08028 Barcelona, Spain.

${ }^{2}$ Institute of Nanoscience and Nanotechnology of the University of Barcelona (IN2UB), Av. Diagonal 645, E-08028 Barcelona, Spain.

${ }^{3}$ Departamento de Física de Materiales e Instituto Pluridisciplinar, Universidad Complutense de Madrid (UCM), 28040 Madrid, Spain.

${ }^{4}$ Departament de Química Inorgànica i Orgànica, Universitat de Barcelona, Martí i Franques 1, E-08028 Barcelona, Spain

${ }^{5}$ Departamento de Ciencias, Universidad Pública de Navarra, 31006 Pamplona, Spain

${ }^{6}$ Institute for Advanced Materials and Mathematics INAMAT, Universidad Pública de Navarra, 31006 Pamplona, Spain.

${ }^{7}$ Catalan Institute of Nanoscience and Nanotechnology (ICN2), CSIC and BIST, Campus UAB, Bellaterra, 08193 Barcelona, Spain.

${ }^{8}$ ICREA, Pg. Lluís Companys 23, E-08010 Barcelona, Spain.

*Corresponding authors: $\quad \underline{\text { ddelpozo@ub.edu, mvarela@ucm.es, }}$ lopezortega.alberto@gmail.com, alejandro.gomez@icn2.cat

\section{Supporting Information}

\section{Methods:}

\section{Nanoparticle synthesis:}

The $\mathrm{FeO} / \mathrm{Fe}_{3} \mathrm{O}_{4}$ core/shell nanocubes were synthesized by thermal decomposition of iron (III) oleate in eicosane. ${ }^{1}$ To synthesize the iron (III) oleate, $16 \mathrm{mmol} \mathrm{FeCl}_{3}(4.32 \mathrm{~g})$ and $14.6 \mathrm{~g}$ sodium oleate $(48 \mathrm{mmol})$ were stirred in a solvent mixture of ethanol $(32 \mathrm{~mL})$, hexane $(56$ $\mathrm{mL})$ and water $(24 \mathrm{~mL})$ and heated up to $79^{\circ} \mathrm{C}$ for 4 hours. Then, the red-brown phase of the reaction mixture was separated washed several times to and finally evaporated under vacuum. In a second reaction, $5.7 \mathrm{mmol}$ iron (III) oleate (5.12 g), $2.5 \mathrm{mmol}$ oleic acid (0.72 g) and 2.6 mmol sodium oleate $(0.78 \mathrm{~g})$ and $7 \mathrm{~g}$ eicosane were mixed and heated up to $100{ }^{\circ} \mathrm{C}$. The reaction system was degassed at $100{ }^{\circ} \mathrm{C}$ for 2 hours under Ar flow. Then, the reaction was heated up to $350{ }^{\circ} \mathrm{C}$ at $3{ }^{\circ} \mathrm{C} / \mathrm{min}$ and kept at this temperature for $30 \mathrm{~min}$. After removing the mixture from the heating source, it was cooled down to room temperature. Finally, the 
nanoparticles were purified by centrifugation with ethanol and hexane several times, and redispersed in n-heptane.

The synthesis of the pure $\mathrm{Fe}_{3} \mathrm{O}_{4}$ nanocubes was carried out through a novel thermal decomposition approach ${ }^{2} .1 .27 \mathrm{mmol}$ iron (III) acetylacetonate $(0.446 \mathrm{~g}), 0.80 \mathrm{mmol}$ sodium oleate $(0.23 \mathrm{~g})$ and $5.20 \mathrm{mmol}$ oleic acid $(1.48 \mathrm{~g})$ were added to a mixture of 1-octadecene (10 $\mathrm{mL})$, dibenzyl ether $(10 \mathrm{~mL})$ and 1-tetradecene $(3 \mathrm{~mL})$. This mixture was heated to $50{ }^{\circ} \mathrm{C}$ and kept at this temperature in vacuum for one hour under vigorous magnetic stirring. Then, the reaction was heated up to $290{ }^{\circ} \mathrm{C}$ at $3{ }^{\circ} \mathrm{C} / \mathrm{min}$ under $\mathrm{Ar}$ flow and kept at this temperature for 60 minutes. Finally, the reaction was cooled to room temperature in Ar flow. The purification of the nanocubes was performed by mixing the product with isopropanol $(50 \mathrm{~mL})$, acetone $(40 \mathrm{~mL})$ and methanol $(10 \mathrm{~mL})$, and centrifuged for 10 minutes at $10600 \mathrm{~g}$, discarding the supernatant. The product was washed again with chloroform $(20 \mathrm{~mL})$, isopropanol $(40 \mathrm{~mL})$ and methanol $(30 \mathrm{~mL})$ and centrifuged again. Finally, the nanoparticles were redispersed in chloroform.

\section{Morphological characterization:}

The morphological characterization was performed in a JEOL JEM-ARM200cF scanning transmission electron microcopy (STEM), operating at $200 \mathrm{kV}$, equipped with a spherical aberration corrector and a cold field emission gun. Annular bright field (ABF) and high angle annular dark field (ADF) STEM operation modes were used to study the sample morphology.

\section{Electron energy-loss spectroscopy (EELS):}

The EEL spectra were acquired in the same STEM instrument equipped with a spherical aberration corrector and a cold field emission gun. The operation voltage used was $200 \mathrm{kV}$ and the energy dispersion used was of $0.25 \mathrm{eV} / \mathrm{ch}$ for data acquisition. Convergence and collection semi-angles of $\alpha=27.8 \mathrm{mrad}$ and $\beta=35 \mathrm{mrad}$ were used, respectively. The beam currents employed were in the tens of $\mathrm{pA}$ range. For the EMCD imaging the illumination settings (such as the condenser lens excitation) were adjusted to obtain a more parallel beam (in the $15-25 \mathrm{mrad}$ range), $0.45 \mathrm{~nm}$ wide, allowing the acquisition of spectrum images at subnanometer spatial resolution. The beam size was additionally estimated from Figure S7. Note that, a Gatan double tilt 636 LN2 sample holder was used to carry out the study at a nominal temperature near $100 \mathrm{~K}$, using liquid nitrogen cooling.

The spectrum images correspond to hyperspectral datasets, typically a data cube, where two of the cube axes correspond to spatial information, $(\mathrm{X}, \mathrm{Y})$, and the third dimension is the electron energy loss spectrum. Visualizing the spectrum image pixel by pixel (X, Y), each spatial position contains an EEL spectrum, and the size of the pixel is related to the probe size of the electron beam. An example of one such spectrum image is given in Figure S8, where the spectrum image of a core/shell nanocube is presented together with two raw spectra, one from the shell, Figure S8b, and another one from the core, Figure S8c. Although in the raw spectra the main spectral features of the oxygen K-edge (around $540 \mathrm{eV}$ ), and the iron white lines $\left(L_{2,3}\right.$-edge; around $\left.710 \mathrm{eV}\right)$ can be identified, there is an important noise component.

To obtain cleaner data, the data were treated in different stages. The first initial basic data treatment was to remove spikes and to correct the energy drift using de HyperSpy library ${ }^{3}$. Secondly, a principal component analysis (PCA) was applied to reduce the noise in the 
spectra and to separate the different components containing relevant information about the iron and oxygen edges ${ }^{4}$.

In addition, to analyze the spectral data, we used the k-means clustering algorithm ${ }^{5}$ to identify different zones in the nanocube and the HyperSpy library ${ }^{3}$. This algorithm uses the energy loss near-edge structures (ELNES) as features to classify the different spectra ${ }^{6}$. The procedure allows to automatically group the spectra by the similarities between them. This algorithm is iterative and groups the data in clusters. Firstly, it identifies the preliminary clusters and computes a mean spectra per cluster. Next, it groups the spectra by resemblances with these mean spectra. Finally, for each cluster the algorithm minimizes the within-cluster variances, classifying each spectrum to the cluster to which it is more similar. This approach makes the spectra classification easier, faster and automatic. In addition, the algorithm also provides the centroid of each cluster, which corresponds to the mean spectrum of all the spectra included in each cluster.

\section{Determination of the oxidation state:}

The Fe oxidation state of the nanoparticles was evaluated using four different EELS methods commonly and widely used for transition metals ${ }^{7-12}$ : (i) the measurement of the $L_{3} / L_{2}$ white line ratio; (ii) the energy distance between the $L$-edge ( $L_{3}$ peak) and the oxygen $K$-edge (main peak); (iii) the energy separation between the $\mathrm{O} K$-edge pre-peak and the main peak and (iv) the ratio between the main peak and the pre-peak of the oxygen $K$-edge.

To calculate the $L_{3} / L_{2}$ ratio [method (i)], first the continuum contribution to the EELS excitation edge was subtracted by a Hartree-Slater cross section. Subsequently, the $L_{3}$ and $L_{2}$ peaks intensities were measured by integrating the remaining intensity in given window widths of $6 \mathrm{eV}$ around the peak maximum. To obtain ratio between the pre-peak and the main peak the oxygen $K$-edge [method (iv)] a similar procedure was applied. Regarding methods (ii) and (iii), the relative energy distances were measured as the energy difference between the center of Gaussian curves best fitting the oxygen $K$-edge and the iron white lines.

Note that all four procedures were carried out pixel-by-pixel for the whole spectrum images. In this way, the errors are minimized identifying the energy value of the edge onsets.

The results are presented as colors maps, where the color scale identifies the iron oxidation state values. References are taken from the values reported in the literature for magnetite and wüstite ${ }^{7,9,13,14}$.

\section{Determination of the elemental composition:}

The elemental quantification of the EELS spectra is obtained using the Digital Micrograph's EELS Quantification tool, utilizing the PCA cleaned spectra and the Hartree-Slater crosssection model.

\section{Electron magnetic circular dichroism (EMCD):}

The EMCD experiments were performed using the EEL spectra acquired as described in the previous section. Note that during the EMCD acquisition the objective lens settings remain unchanged, implying the presence of a large magnetic field of 2-3 $\mathrm{T}$ within the pole piece.

The EELS data for the EMCD experiment were acquired using the intrinsic method described by Rubino et al. ${ }^{15}$ and Salafranca et al. ${ }^{16}$, in which the crystalline sample is tilted to obtain 
two-beam conditions and the splitting of the beam is achieved via Bragg scattering. In our case, the transmitted beam and the Bragg spot $\vec{g}=\left(\begin{array}{lll}2 & 2 & 0\end{array}\right)$ were used. Considering this scattering geometry, the dichroic signal was obtained from the intensity difference in the iron white lines $\left(L_{3}\right.$ and $\left.L_{2}\right)$ between two spectra acquired from the same region of the nanocubes, placing the spectrometer entrance aperture (SEA) of the EELS detector in two symmetric positions, namely, in the ' + ' or right circular polarization (A) and the '-' or left circular polarization (B), as shown in Figure S9.

Apart from the noise reduction methods applied to the EELS data, an additional correction was applied to the EMCD data to correct for small deviations of the energy positions in the elastic peaks (a few eV) induced by the different tilting of the SEA. Namely, the energy of the spectra was manually aligned considering the oxygen $K$-edge pre-peak as a fixed energy value at $530 \mathrm{eV}$. This particular energy was chosen since the pre-peak onset remains almost the same for magnetite and wüstite ${ }^{7,9,14}$.

To be able to calculate the dichroic signal, for all the spectra the pre-edge background of the iron white lines was removed using a power-law background fit to the region between 670$690 \mathrm{eV}$ and a post-edge normalization was implemented considering the region between 730$760 \mathrm{eV}$. This last normalization was required to remove the continuum contribution and faithfully calculate the dichroic signals ${ }^{17}$.

In order to be able to compare the dichroic signals obtained from the different core/shell samples, it is necessary to normalize the EMCD signal. Thus, for that purpose the area of the experimental absorption spectra (Spectra ${ }_{+}+$Spectra $\left._{-}\right)$was considered as a normalization factor $^{18}$. Hence, the normalized intensity of EMCD signal can be calculated as:

$$
I_{E M C D}=\frac{\text { Spectra }_{+}-\text {Spectra }_{-}}{A_{\text {Spectra }}+\text { Spectra }}-
$$

where $A_{\text {Spectra }}+$ Spectra $a_{-}$was obtained by integrating the sum of the two spectra from 702 to $739 \mathrm{eV}$.

To obtain semi-quantitative information from the EMCD signals, the area of the $L_{3}-L_{2}$ peaks in the EMCD spectrum was computed. Namely, a mathematical integration (difference between positive and negative areas) of the EMCD signal considering the integration limits from 702 to $739 \mathrm{eV}$, including the white lines. The error was calculated measuring the area at the pre-edge (670 to $700 \mathrm{eV}$ ), where there was no magnetic contribution.

\section{Magnetic characterization:}

The bulk magnetic characterization was carried out using a superconducting quantum interference device (SQUID, Quantum Design) magnetometer. The temperature dependence of magnetization, $\mathrm{M}(\mathrm{T})$, was measured during warming using an applied filed of $50 \mathrm{Oe}$, after either zero field cooling (ZFC) or field cooling (FC) in 50 Oe from $300 \mathrm{~K}$ to $10 \mathrm{~K}$. 


\section{Complementary Figures:}

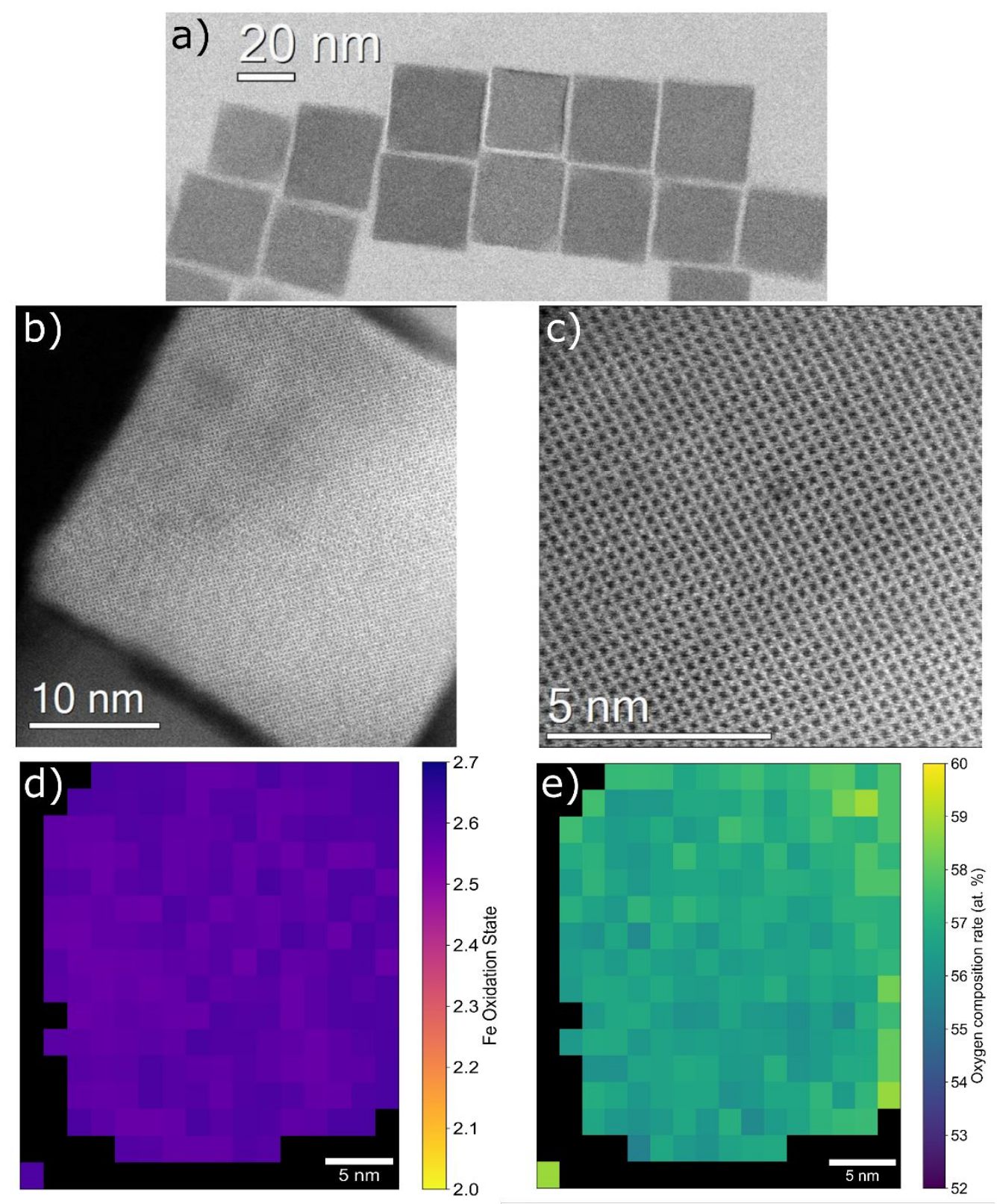

Figure S1. (a) Bright Field (BF) STEM image at low magnification of pure magnetite nanocubes, (b) Annular Dark Field (ADF) STEM image of an individual pure magnetite nanocube, and (c) ADF STEM image taken at high magnification of a pure magnetite nanocube. (d) Color maps of the iron oxidation state of a $\mathrm{Fe}_{3} \mathrm{O}_{4}$ nanocube obtained from the $\mathrm{L}_{3} / \mathrm{L}_{2}$ ratio (method $\mathrm{i}$; see Methods section). (e) Color map of the oxygen elemental composition rate at atomic percent (at. \%). 


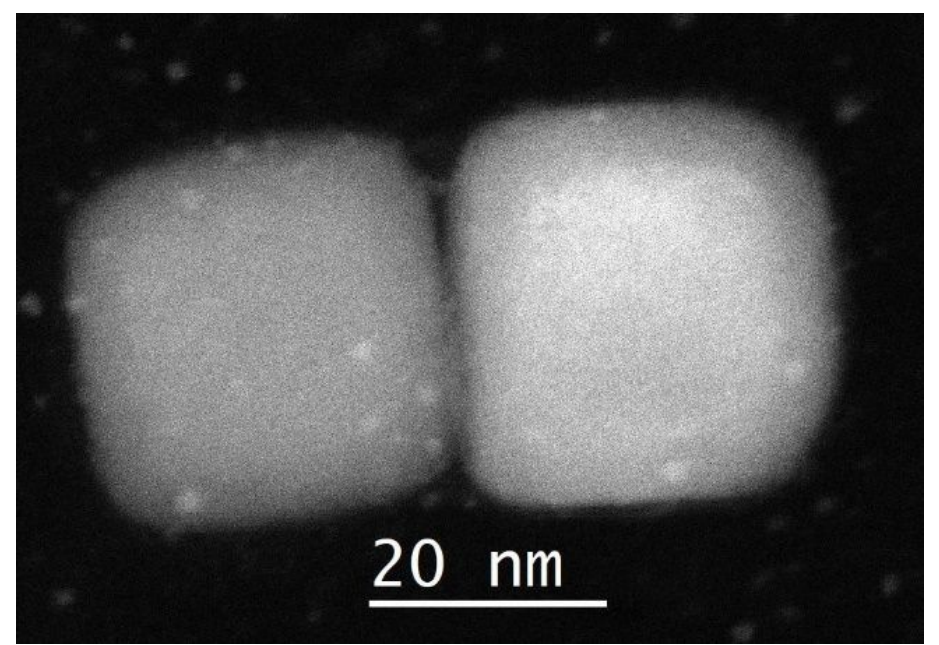

Figure S2. Lower magnification STEM high angle annular dark field image of the core/shell nanocube presented in Figures 2 and 3, which corresponds to the one at right-hand side of this image. 

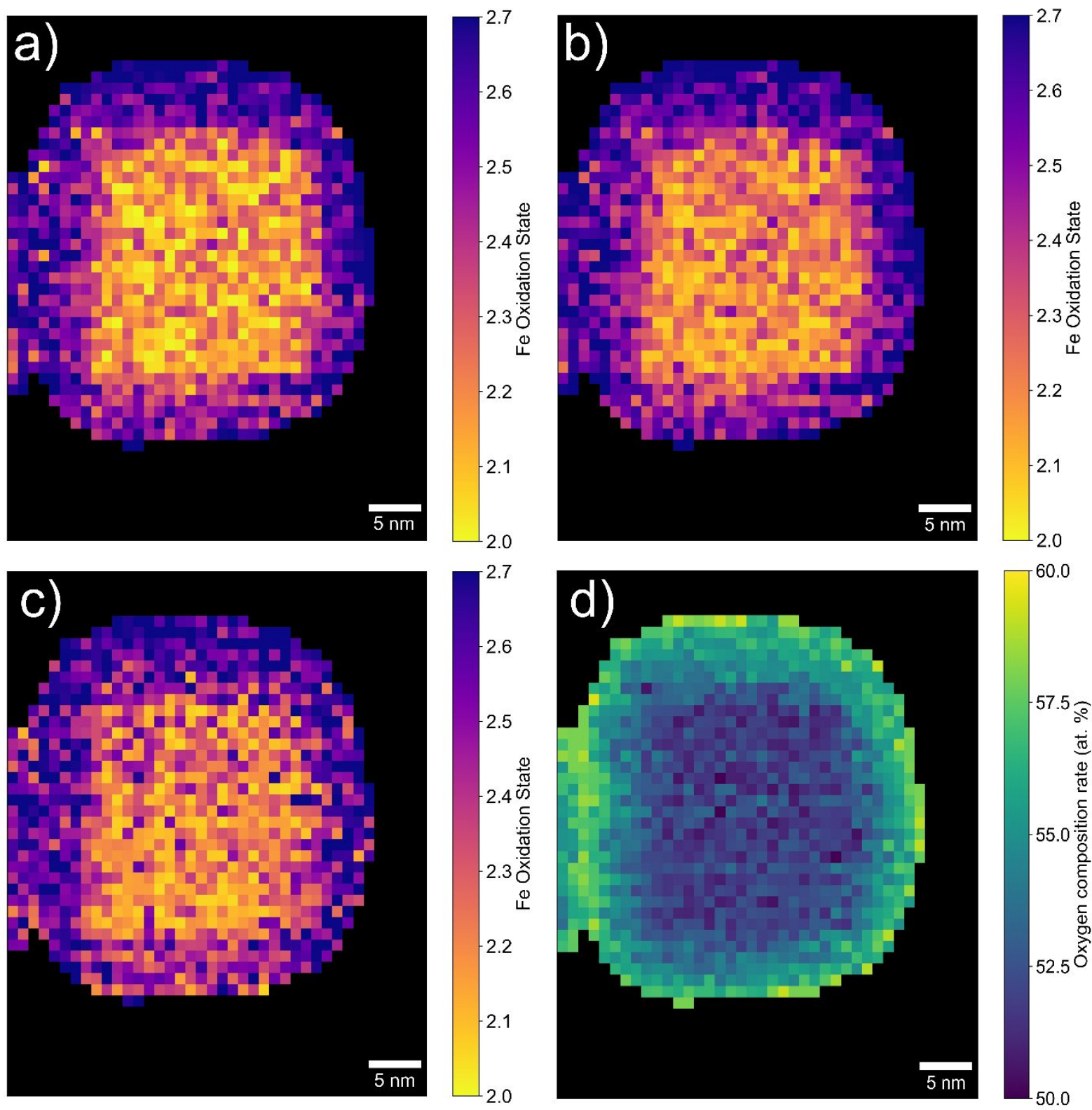

Figure S3. Color maps of the iron oxidation state obtained from: (a) the $\mathrm{L}_{3} / \mathrm{L}_{2}$ ratio [method i], (b) energy separation between the O K-edge pre-peak and main peak [method iii], and (c) the relative intensity of the oxygen $\mathrm{K}$ pre-peak with respect to the main $\mathrm{O} \mathrm{K}$-edge [method iv]. (d) Color map of the oxygen elemental composition rate at atomic percent (at. \%). 


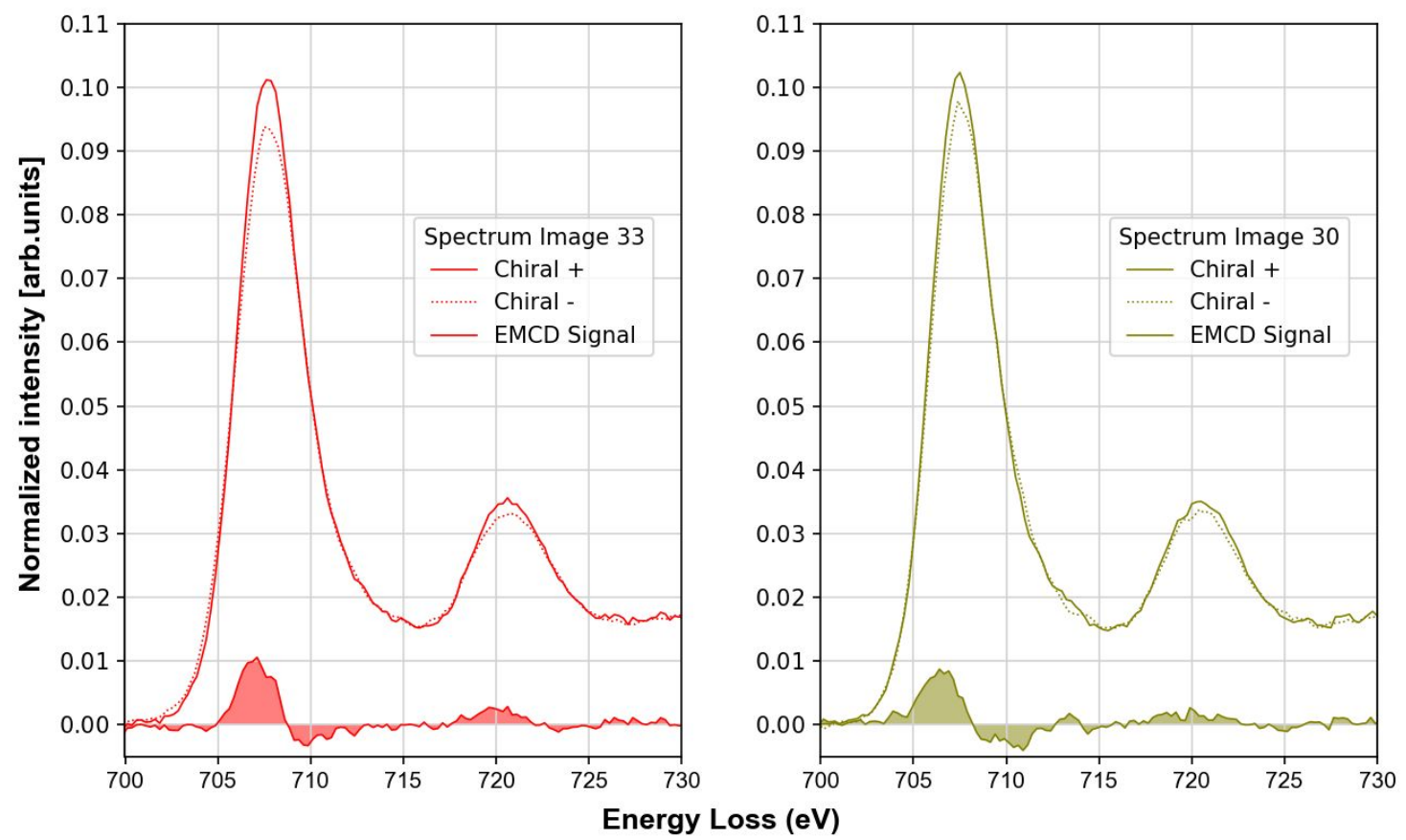

Figure S4. Spectra of the + and - chiralities and the corresponding EMCD signal for two different pure $\mathrm{Fe}_{3} \mathrm{O}_{4}$ nanocubes.

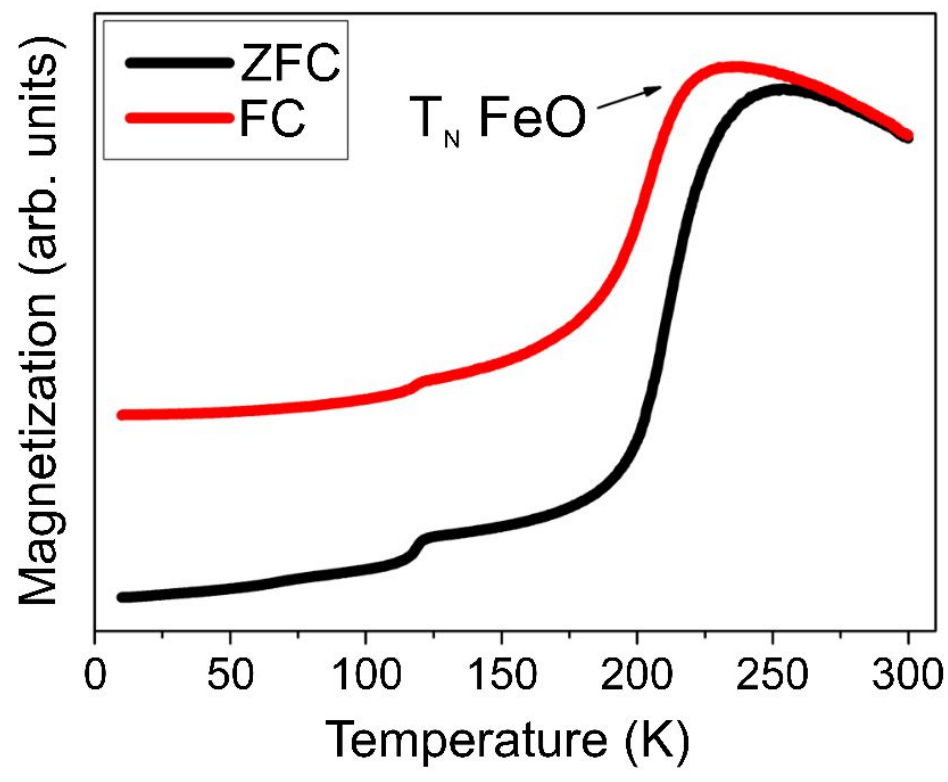

Figure S5. Field cooled (FC) and zero field cooled (ZFC) magnetization of the $\mathrm{FeO} / \mathrm{Fe}_{3} \mathrm{O}_{4}$ core/shell nanocubes at $\mathrm{H}=50 \mathrm{Oe}$. The Néel temperature, $\mathrm{T}_{\mathrm{N}}$, of the $\mathrm{FeO}$ phase is highlighted by an arrow. 

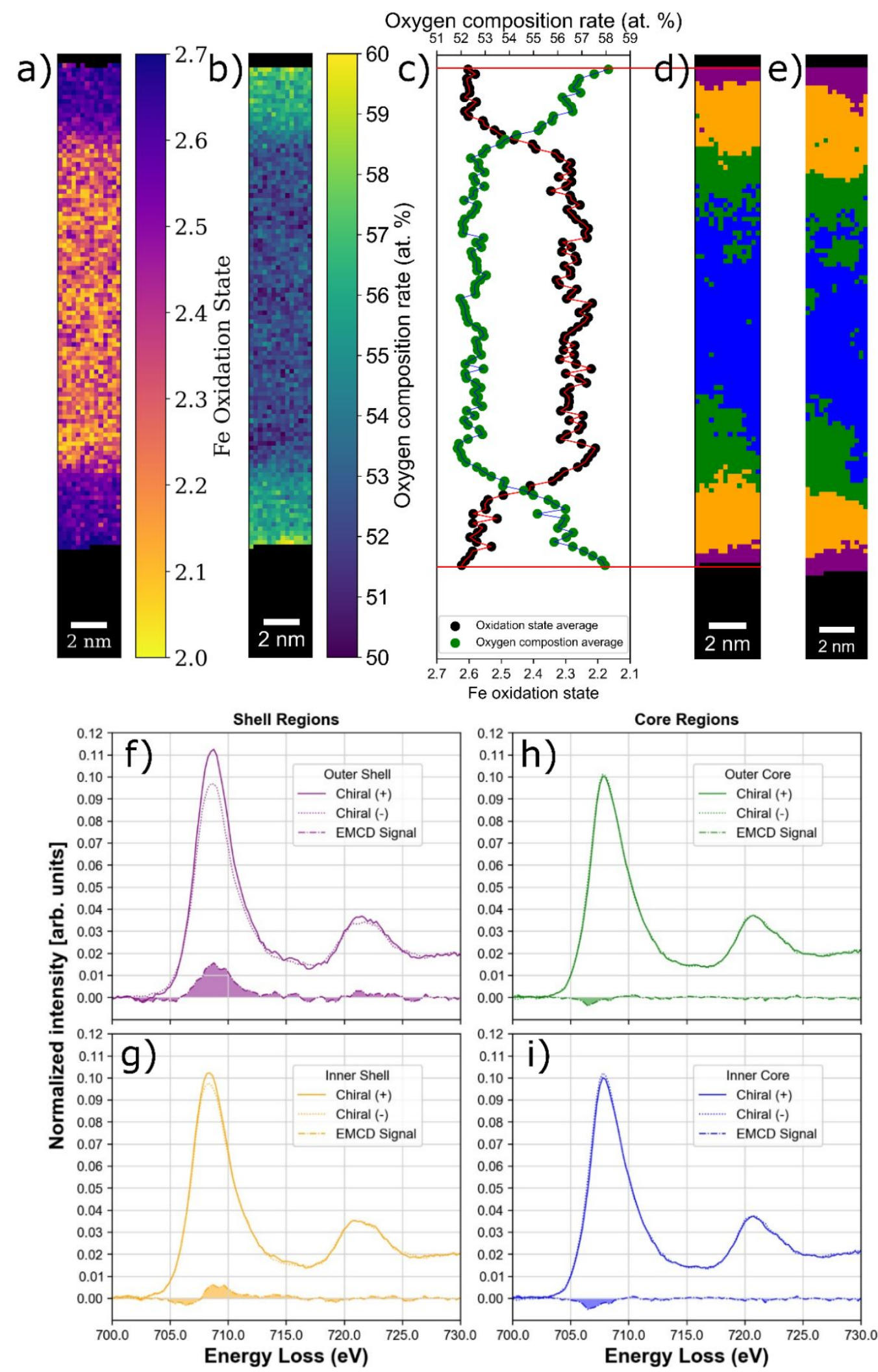

Figure S6. (a) Color map of the iron oxidation state of a $\mathrm{FeO} / \mathrm{Fe}_{3} \mathrm{O}_{4}$ core/shell nanocube section obtained from the distance between the oxygen $\mathrm{K}$ edge and the $\mathrm{L}_{3}$ iron white line (method ii, see Methods section). (b) Color map of the oxygen elemental quantification at atomic percent (at. \%) of a $\mathrm{FeO} / \mathrm{Fe}_{3} \mathrm{O}_{4}$ core/shell nanocube. (c) Line profiles of the averaged oxidation state (black dots) and the oxygen elemental composition rate (green dots) (averaged horizontally in both cases, and in the oxidation case for the four analysis methods). (d, e) Color maps resulting from the clustering analysis for (d) chiral (+) and (e) chiral (-) configuration of a core/shell nanocube. (f-i) Spectra of the + and -

chiralities for each section identified in the clustering and the corresponding EMCD signal for a $\mathrm{FeO} / \mathrm{Fe} 3 \mathrm{O} 4$ core/shell nanocube: (f) outer shell (violet); (g) inner shell (orange); (h) outer core (green) and (i) inner core (blue). 


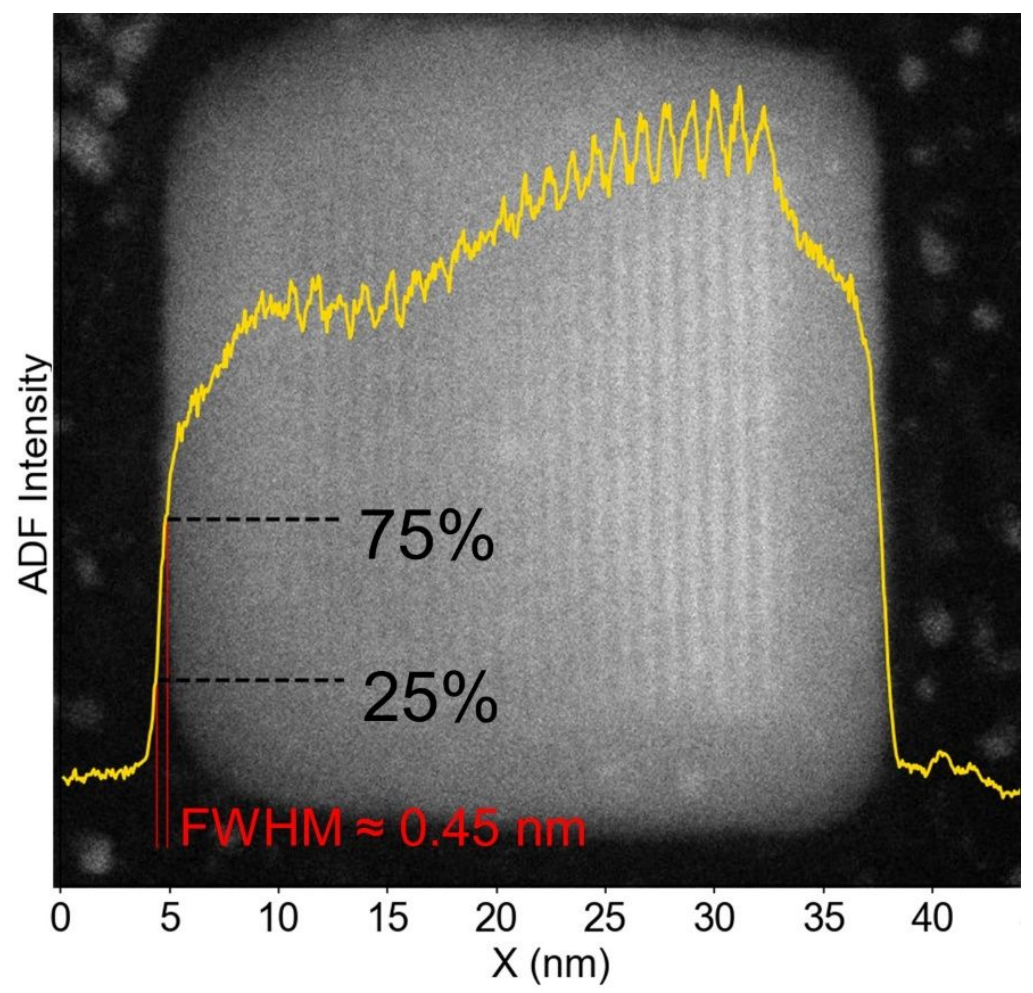

Figure S7. ADF image of a $30 \mathrm{~nm}$ sized core/shell nanocube. Superimposed is the ADF signal profile. Assuming a Gaussian beam, the beam FWHM can be estimated as the width between $25 \%$ and $75 \%$ of the ADF intensity drop at the edge.
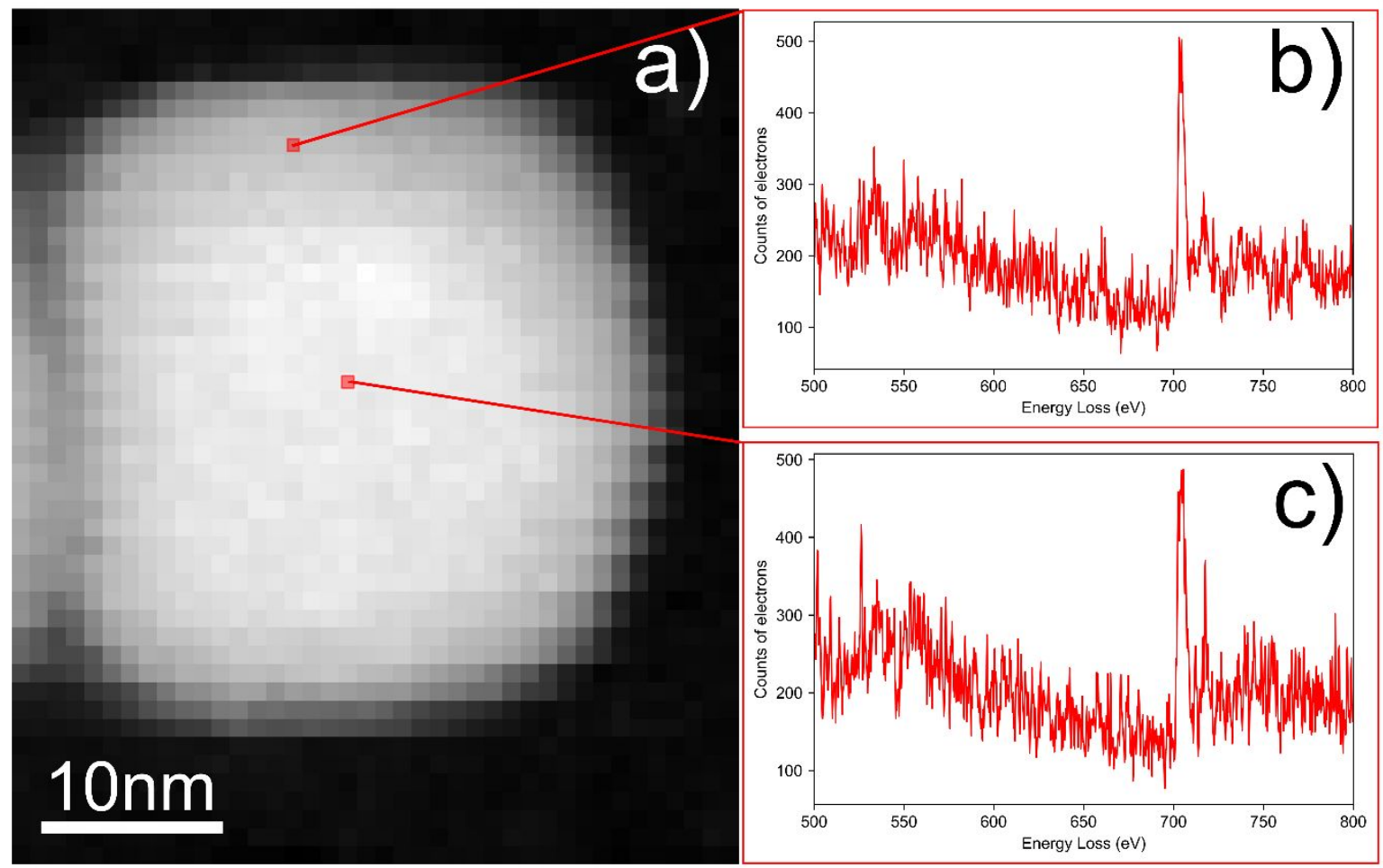

Figure S8. (a) Spectrum image of the original data from a chiral $(+)$ configuration. Original energy loss spectra: (b) from the magnetite shell, and (c) from the wüstite core. 


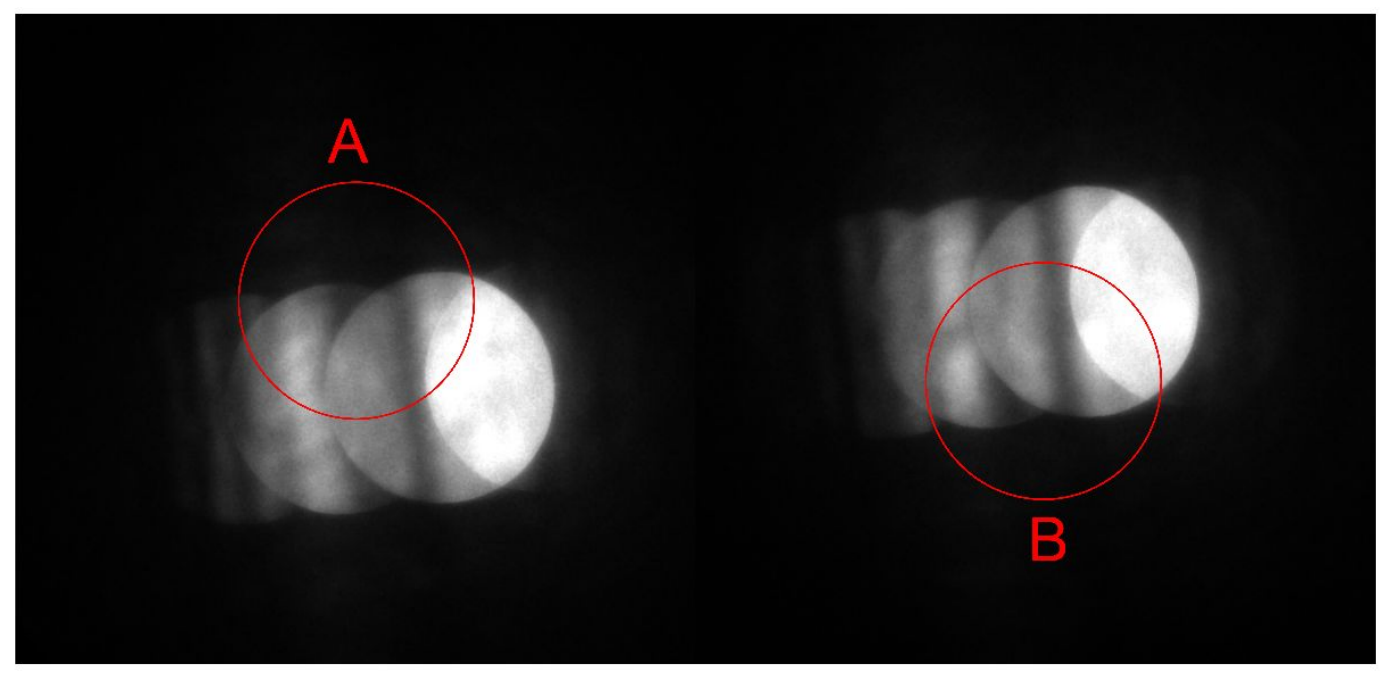

Figure S9. A and B positions of the spectrometer entrance aperture (SEA) for the EMCD experiments. Position A corresponds to the chiral (+) and position B to the chiral (-). These experimental configurations correspond to EMCD experiment of the core/shell nanocube in Figure 2.

\section{References:}

(1) Estrader, M.; López-Ortega, A.; Golosovsky, I. V.; Estradé, S.; Roca, A. G.; Salazar-Alvarez, G.; López-Conesa, L.; Tobia, D.; Winkler, E.; Ardisson, J. D.; Macedo, W. A. A.; Morphis, A.; Vasilakaki, M.; Trohidou, K. N.; Gukasov, A.; Mirebeau, I.; Makarova, O. L.; Zysler, R. D.; Peiró, F.; Baró, M. D.; Bergström, L.; Nogués, J. Origin of the Large Dispersion of Magnetic Properties in Nanostructured Oxides: $\mathrm{Fe}_{\mathrm{x}} \mathrm{O} / \mathrm{Fe}_{3} \mathrm{O}_{4}$ Nanoparticles as a Case Study. Nanoscale 2015, 7, 3002-3015. https://doi.org/10.1039/C4NR06351A.

(2) Muro-Cruces, J.; Roca, A. G.; López-Ortega, A.; Fantechi, E.; Del-Pozo-Bueno, D.; Estradé, S.; Peiró, F.; Sepúlveda, B.; Pineider, F.; Sangregorio, C.; Nogues, J. Precise Size Control of the Growth of $\mathrm{Fe}_{3} \mathrm{O}_{4}$ Nanocubes over a Wide Size Range Using a Rationally Designed One-Pot Synthesis. ACS Nano 2019, 13, 7716-7728. https://doi.org/10.1021/acsnano.9b01281.

(3) Peña, F. de la; Prestat, E.; Fauske, V. T.; Burdet, P.; Furnival, T.; Jokubauskas, P.; Lähnemann, J.; Nord, M.; Ostasevicius, T.; MacArthur, K. E.; Johnstone, D. N.; Sarahan, M.; Taillon, J.; Aarholt, T.; Migunov, V.; Eljarrat, A.; Caron, J.; Poon, T.; Mazzucco, S.; Martineau, B.; Somnath, S.; Slater, T.; Francis, C.; Tappy, N.; Walls, M.; Cautaerts, N.; Winkler, F.; Donval, G. Hyperspy/Hyperspy: Release v1.6.4. 2021. https://doi.org/10.5281/ZENODO.5082777.

(4) Cueva, P.; Hovden, R.; Mundy, J. A.; Xin, H. L.; Muller, D. A. Data Processing for Atomic Resolution Electron Energy Loss Spectroscopy. Microsc. Microanal. 2012, 18, 667-675. https://doi.org/10.1017/S1431927612000244.

(5) Torruella, P.; Estrader, M.; López-Ortega, A.; Baró, M. D.; Varela, M.; Peiró, F.; Estradé, S. Clustering Analysis Strategies for Electron Energy Loss Spectroscopy (EELS). Ultramicroscopy 2018, 185, 42-48. https://doi.org/10.1016/j.ultramic.2017.11.010.

(6) Torruella, P.; Arenal, R.; De La Peña, F.; Saghi, Z.; Yedra, L.; Eljarrat, A.; López-Conesa, L.; Estrader, M.; López-Ortega, A.; Salazar-Alvarez, G.; Nogués, J.; Ducati, C.; Midgley, P. A.; Peiró, F.; Estradé, S. 3D Visualization of the Iron Oxidation State in $\mathrm{FeO} / \mathrm{Fe}_{3} \mathrm{O}_{4}$ Core-Shell Nanocubes from Electron Energy Loss Tomography. Nano Lett. 2016, 16, 5068-5073. https://doi.org/10.1021/acs.nanolett.6b01922.

(7) Colliex, C.; Manoubi, T.; Ortiz, C. Electron-Energy-Loss-Spectroscopy near-Edge Fine Structures in the Iron-Oxygen System. Phys. Rev. B: Condens. Matter Mater. Phys. 1991, 44, 11402-11411. https://doi.org/10.1103/PhysRevB.44.11402. 
(8) Varela, M.; Oxley, M. P.; Luo, W.; Tao, J.; Watanabe, M.; Lupini, A. R.; Pantelides, S. T.; Pennycook, S. J. Atomic-Resolution Imaging of Oxidation States in Manganites. Phys. Rev. B: Condens. Matter Mater. Phys. 2009, 79, 085117. https://doi.org/10.1103/PhysRevB.79.085117.

(9) Tan, H.; Verbeeck, J.; Abakumov, A.; van Tendeloo, G. Oxidation State and Chemical Shift Investigation in Transition Metal Oxides by EELS. Ultramicroscopy 2012, 116, 24-33. https://doi.org/10.1016/j.ultramic.2012.03.002.

(10) Yedra, L.; Xuriguera, E.; Estrader, M.; López-Ortega, A.; Baró, M. D.; Nogués, J.; Roldan, M.; Varela, M.; Estradé, S.; Peiró, F. Oxide Wizard: An EELS Application to Characterize the White Lines of Transition Metal Edges. Microsc. Microanal. 2014, 20, 698-705. https://doi.org/10.1017/S1431927614000440.

(11) Arévalo-López, Á. M.; Alario-Franco, M. Á. Reliable Method for Determining the Oxidation State in Chromium Oxides. Inorg. Chem. 2009, 48, 11843-11846. https://doi.org/10.1021/ic901887y.

(12) Luo, K.; Roberts, M. R.; Hao, R.; Guerrini, N.; Pickup, D. M.; Liu, Y. S.; Edström, K.; Guo, J.; Chadwick, A. v.; Duda, L. C.; Bruce, P. G. Charge-Compensation in 3d-Transition-MetalOxide Intercalation Cathodes through the Generation of Localized Electron Holes on Oxygen. Nat. Chem. 2016, 8, 684-691. https://doi.org/10.1038/nchem.2471.

(13) van Aken, P. A.; Liebscher, B. Quantification of Ferrous/Ferric Ratios in Minerals: New Evaluation Schemes of $\mathrm{Fe}_{23}$ Electron Energy-Loss near-Edge Spectra. Phys. Chem. Miner. 2002, 29, 188-200. https://doi.org/10.1007/s00269-001-0222-6.

(14) Calvert, C. C.; Brown, A.; Brydson, R. Determination of the Local Chemistry of Iron in Inorganic and Organic Materials. J. Electron Spectrosc. Relat. Phenom. 2005, 143, 173-187. https://doi.org/10.1016/j.elspec.2004.03.012.

(15) Rubino, S.; Schattschneider, P.; Stöger-Pollach, M.; Hébert, C.; Rusz, J.; Calmels, L.; WarotFonrose, B.; Houdellier, F.; Serin, V.; Novak, P. Energy-Loss Magnetic Chiral Dichroism (EMCD): Magnetic Chiral Dichroism in the Electron Microscope. J. Mater. Res. 2008, 23, 2582-2590. https://doi.org/10.1557/jmr.2008.0348.

(16) Salafranca, J.; Gazquez, J.; Pérez, N.; Labarta, A.; Pantelides, S. T.; Pennycook, S. J.; Batlle, X.; Varela, M. Surfactant Organic Molecules Restore Magnetism in Metal-Oxide Nanoparticle Surfaces. Nano Lett. 2012, 12, 2499-2503. https://doi.org/10.1021/nl300665z.

(17) Thersleff, T.; Rusz, J.; Rubino, S.; Hjörvarsson, B.; Ito, Y.; Zaluzec, N.; Leifer, K. Quantitative Analysis of Magnetic Spin and Orbital Moments from an Oxidized Iron (1 110$)$ Surface Using $\begin{array}{lllll}\text { Electron Magnetic Circular Dichroism. Sci. } & \text { Rep. } & \mathbf{2 0 1 5}, & 5,\end{array}$ https://doi.org/10.1038/srep13012.

(18) Wang, Z. Q.; Zhong, X. Y.; Yu, R.; Cheng, Z. Y.; Zhu, J. Quantitative Experimental Determination of Site-Specific Magnetic Structures by Transmitted Electrons. Nat. Commun. 2013, 4, 1395. https://doi.org/10.1038/ncomms2323. 